\title{
Filigrane
}

Écoutes psychanalytiques

\section{L'actualité de la psychanalyse : de sa place dans l'institution du soin aux dispositifs cliniques}

\section{Nadine Proia-Lelouey}

Volume 24, numéro 2, automne 2015

Le devenir de la psychanalyse. Que nous disent les pratiques institutionnelles?

URI : https://id.erudit.org/iderudit/1036533ar

DOI : https://doi.org/10.7202/1036533ar

Aller au sommaire du numéro

\section{Éditeur(s)}

Revue Santé mentale au Québec

ISSN

1192-1412 (imprimé)

1911-4656 (numérique)

Découvrir la revue

Citer cet article

Proia-Lelouey, N. (2015). L’actualité de la psychanalyse : de sa place dans l'institution du soin aux dispositifs cliniques. Filigrane, 24(2), 93-107.

https://doi.org/10.7202/1036533ar

\section{Résumé de l'article}

L'article pose sur deux registres différents mais interdépendants la place de la psychanalyse dans le soin aujourd'hui. Il s'agit dans un premier temps de montrer, en se référant à des analyses sociologiques, que cette question est étroitement liée aux différentes manières d'appréhender la pathologie mentale au cours du 20e siècle. Evolution elle-même liée à des changements de la condition anthropologique du sujet. Ainsi, si la psychanalyse a été au coeur de l'institution du soin pour avoir largement contribué dans les années 1950/1960 au passage du paradigme de la pathologie mentale à celui de la souffrance psychique, elle a, en revanche, largement pâti d'une rupture qui, au cours des années 1980/1990, a mis au centre du soin le paradigme normatif de la santé mentale. La psychanalyse devient alors une forme de résistance afin que la norme n'efface pas totalement le sujet dans les institutions de soins. Mais la psychanalyse doit se transformer et penser de nouveaux dispositifs à partir des nouvelles conditions anthropologiques du sujet. L'auteure soutient que l'enjeu fondamental de cette nouvelle psychanalyse concerne la fonctionnalité du préconscient et l'accès à la capacité réflexive. Elle développe cette réflexion à partir des travaux contemporains engagés par R. Kaës et R. Roussillon sur l'analyse transitionnelle.
Ce document est protégé par la loi sur le droit d'auteur. L'utilisation des services d’Érudit (y compris la reproduction) est assujettie à sa politique d'utilisation que vous pouvez consulter en ligne.

https://apropos.erudit.org/fr/usagers/politique-dutilisation/ 


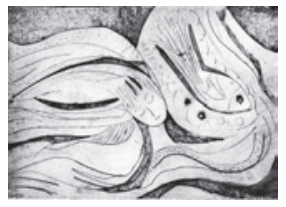

\section{L'actualité de la psychanalyse: de sa place dans l'institution du soin aux dispositifs cliniques}

Nadine Proia-Lelouey

L'article pose sur deux registres différents mais interdépendants la place de la psychanalyse dans le soin aujourd'hui. Il s'agit dans un premier temps de montrer, en se référant à des analyses sociologiques, que cette question est étroitement liée aux différentes manières d'appréhender la pathologie mentale au cours du 20e siècle. Evolution elle-même liée à des changements de la condition anthropologique du sujet. Ainsi, si la psychanalyse a été au cœur de l'institution du soin pour avoir largement contribué dans les années 1950/1960 au passage du paradigme de la pathologie mentale à celui de la souffrance psychique, elle a, en revanche, largement pâti d'une rupture qui, au cours des années 1980/1990, a mis au centre du soin le paradigme normatif de la santé mentale. La psychanalyse devient alors une forme de résistance afin que la norme n'efface pas totalement le sujet dans les institutions de soins. Mais la psychanalyse doit se transformer et penser de nouveaux dispositifs à partir des nouvelles conditions anthropologiques du sujet. L'auteure soutient que l'enjeu fondamental de cette nouvelle psychanalyse concerne la fonctionnalité du préconscient et l'accès à la capacité réflexive. Elle développe cette réflexion à partir des travaux contemporains engagés par R. Kaës et R. Roussillon sur l'analyse transitionnelle.

\section{u'est la psychanalyse devenue? État des lieux auquel se livre Filigrane depuis quelque temps. Nous avons entendu cette question comme une manière de s'interroger sur les conditions actuelles de l'exercice de la psy- chanalyse dans les institutions de soins. Question d'importance au regard du déchaînement d'hostilité dont elle est l'objet (Roudinesco, 2005). Nous ne citerons, à titre d'illustration, qu'une attaque très récente et pour le moins surprenante. Celle de Benasayag (2015) qui balaie quasiment un siècle de pratique psychanalytique institutionnelle considérant celle-ci, dans une vision fort caricaturale, comme inapte à prendre en charge les probléma- tiques cliniques contemporaines. \\ Après avoir été portée aux nues (Castel, 1973), elle serait donc la pire des pratiques: ringarde, inefficace, non scientifique, onéreuse. Discours aussi caricatural que son pendant durant les années 1950-1970 qui en faisait la}


référence absolue et la réponse à tout, bien au-delà du soin (sciences sociales, culture, etc.).

Face à de tels discours, il devient en effet urgent d'élaborer notre positionnement afin de répondre sans acrimonie ni polémique à nos détracteurs. Mon propos portera sur deux points: dans un premier temps, je souhaite interroger la place de la psychanalyse dans l'institution $d u$ soin; dans un second temps je voudrais aborder les conséquences, sur les dispositifs cliniques, des évolutions constatées dans le champ institutionnel.

Évoquer l'exercice psychanalytique dans les institutions du soin peut s'entendre de deux manières. Une première serait de réfléchir sur les dispositifs psychanalytiques. C'est ce que nous ferons dans un second temps en nous référant à l'analyse transitionnelle (Kaës, 2012; Roussillon, 2008). Mais cette approche doit être éclairée par une autre qu’il est nécessaire de traiter en amont: quelle place pour la psychanalyse dans l'institution $d u$ soin? Poser la question de cette manière c'est s'inscrire dans une perspective durkheimienne de l'institution. On distingue en effet classiquement deux approches de l'institution: celles d'Émile Durkheim et de Max Weber (Proia-Lelouey, 2012). Selon la première, "l'institution constitue un ensemble de dispositions et d'aménagements qui sont culturellement et socialement construits autour d'une activité sociale et qui tendent à se perpétuer dans l'espace et le temps.» (Turmel, 1997, 13). La seconde relève en réalité de l'organisationnel (Juan, 2006). Les individus ont besoin d'inscrire, d'organiser leurs institutions (au sens de Durkheim) dans des structures, des organisations. Si les deux niveaux sont interdépendants, le premier reste déterminant car il fixe le cadre dans lequel vont être opérationnalisés les dispositifs de soin mis en place par les organisations du soin. Ce processus rend compte de comment la dynamique institué/instituant vient s'exprimer dans l'organisationnel.

Indubitablement, la psychanalyse a largement fait partie de cette dynamique institué/instituant; elle est donc au cour de l'institution du soin. Nous en retiendrons pour preuve les analyses d'abord de R. Castel (1973) et de R. Castel et J. F. Le Cerf (1980a, 1980b, 1980c), puis d'A. Ehrenberg (2010), qui attribuent à la psychanalyse la diffusion du psychologique dans le champ social du fait de son explosion médiatique dans l'après-guerre. Il semblerait plus juste de tenir le discours inverse (ou tout au moins d'en faire un processus systémique) et de dire que le succès de la psychanalyse tient à la diffusion du concept d'individualité dans le discours social. Comme A. Ehrenberg l'écrit lui-même: « une nouvelle culture à la fois du bonheur et de l'action se diffuse dans l'hexagone au moment où se produit une valorisation 
de la vie privée vers $1960 »$ (p. 177). Ainsi la psychanalyse serait venue offrir un langage dans lequel pouvait s'exprimer une nouvelle manière d'être dans le monde. L'auteur donne d'ailleurs (p. 173-188) les deux raisons du succès de la psychanalyse: développement d'un langage de l'intériorité par le biais des médias, certes alimenté par une vulgate psychanalytique; développement du soin psychique pour une population toujours plus large (p. 242). Les états d'âme - comme le temps oisif, apanage de la société bourgeoise du XIX $X^{e}$ siècle - se démocratisent et sont mis à la portée de tous (congés payés et centres de consultation public). Phénomène qui débute dans l'entredeux-guerres et se développe considérablement à partir des années 1950.

Mais paradoxalement, en s'ouvrant à un large public et en se faisant le relais de l'aspiration à l'autonomie (voir ci-dessous), la psychanalyse, toujours selon A. Ehrenberg, trouve ses limites et se voit interrogée dans ce qui la fonde (l'CEdipe, la cure). Elle en vient à se décentrer de la névrose pour se focaliser sur la dépression, à admettre un triomphe de Narcisse sur CEdipe et à conceptualiser de nécessaires aménagements de la cure. Il s'agit là de tout l'apport des psychanalystes de l'après-guerre à la question des sujets limites que Freud, au seuil de sa vie, avait amorcée (1940/2010, p. 266).

Si A. Ehrenberg admet le constat de cette évolution de la clinique, il est très critique sur l'analyse des causes de cette évolution telle qu'elle peut être faite par les psychanalystes. Selon ces derniers, l'extension des pathologies du narcissisme serait la résultante d'une mutation socio-anthropologique ${ }^{1}$ qui mettrait à mal le (Père-)Symbolique, les Institutions ${ }^{2}$ et/ou encore la civilisation. Analyses inspirées des travaux de M. Gauchet $(1998,2002)$ et relayées par des analyses sociologiques. L'auteur cite C. Lasch (1981) aux États-Unis ou G. Lipovetsky (1983) en France ${ }^{3}$.

Sans entrer dans le détail du débat qui l'oppose à ces auteurs, nous retiendrons avec A. Ehrenberg, qu'il s'agit moins d'une perte du symbolique, d'une rupture dans le contrat social que d'une nouvelle manière de faire société et que celle-ci passe par l'individuel et donc le psychologique. Il évoque les travaux de R. Castel (1981) et précise que selon ce dernier: «la psychologisation signifie donc moins l'absorption en soi de Narcisse que l'instrumentalisation de la subjectivité individuelle qui dispensera de réformer la société» (Ehrenberg, 2010, p. 210). L'auteur $(1995,2010)$ poursuit ainsi le programme initié par $\mathrm{R}$. Castel visant à mener une réflexion critique sur la subjectivité libérée et instrumentalisée. Il précise que cette nouvelle manière de faire société passe par l'autonomie des individus (2010, p. 189219). Nous ne serions plus alors dans un contrat social basé sur l'obligation, 
la contrainte ( fais ceci, ne fais pas cela»), mais basé sur la responsabilité («tu es libre mais tu es comptable des résultats»). L'auteur distingue par ailleurs deux types d'autonomie: l'autonomie comme aspiration et l'autonomie comme condition, situant la bascule autour des années 1980: «l'autonomie non au sens d'une liberté de participation accrue à la décision politique, mais un changement dans l'esprit de l'action qui fait de l'autonomie des individus, de la capacité à se gouverner par soi-même et à agir de soi-même la valeur suprême» (2010, p. 213-214).

On peut considérer, même si l'auteur n'apprécie guère cette terminologie, qu'il évoque, avec les notions d'obligation, d'aspiration à l'autonomie et d'autonomie comme condition, les enjeux de l'homme moderne, postmoderne et hypermoderne. Bien entendu, il n'est pas question ici d'évoquer trois types cliniques, mais trois conditions de la subjectivation en lien avec la nature du contrat social, sachant que nous envisageons ces trois modalités comme non exclusives les unes des autres.

Que devient l'institution du soin (et la psychanalyse) dans ces nouveaux enjeux?

Freud fait le lien entre psychopathologie individuelle et culture (contrat social) en posant une opposition inévitable entre exigences de la pulsion et le nécessaire vivre ensemble. Il considère ce conflit comme indépassable, car si le but de l'individu est le bonheur, le but de la culture est «d'instaurer une unité à partir des individus» (1930/1994, p. 328) quitte à y sacrifier une part du bonheur de chacun.

Analysant la société moderne, il fait donc du contrat social par obligation une source de souffrance psychique. Il fixe alors comme tâche au thérapeute d'en assouplir les effets: "Ainsi sommes-nous très souvent obligés, dans une visée thérapeutique, de combattre le sur-moi et nous nous efforçons de rabattre ses revendications» (Freud, 1930/1994, p. 330).

Mais dans ce texte, Freud met surtout l'accent sur les pulsions destructrices et leur nécessaire répression. S'agit-il alors pour lui de préconiser davantage de libido pour mieux lier la pulsion destructrice? De lutter contre une alliance entre pulsion de mort et surmoi (p. $310^{4}$ ) au profit de davantage de satisfaction libidinale? Il considère par ailleurs qu'il y a un redoublement entre surmoi-de-la-culture et surmoi individuel (p. 329), et donc que face à la répression du premier, il faut assouplir le second.

Mais dans un discours qui s'inverse, dans une culture permissive, le surmoi-de-la-culture ne fait plus son œuvre. Au contraire, il exacerbe les exigences de satisfaction pulsionnelle. L'homme postmoderne, en accord avec 
le nouveau contrat social, réclame sa part de satisfaction et de bonheur. $\mathrm{Ne}$ pouvant plus accepter la passagèreté de la jouissance (Freud, 1916/1988, p. 323), il déprime ou s'adonne aux Paradis artificiels (Ehrenberg, 1998).

En contrepoint, l'institution du soin devient le lieu de plaintes sur ce bonheur, ce plaisir de droit qui pourtant ne vient pas... la faisant passer, dans un premier glissement, du paradigme de la pathologie à celui de la souffrance psychique. Ainsi, pour pallier le leurre induit par la culture postmoderne, les individus se voient offrir un droit à l'écoute, nouveau fondement de l'institution du soin. Celui-ci est davantage référé, dans sa réalité, au courant humaniste à la C. Rogers qu'au courant psychanalytique (Ehrenberg, 2010, p. 177) comme l'écrivaient déjà R. Castel et J. F. Le Cerf: «Elle [la psychanalyse] a été le principal instrument de diffusion d'une nouvelle culture psychologique dans laquelle, aujourd'hui, elle commence à se perdre» (1980a, p. 33). Sa constellation symbolique pourrait être: expression de soi, écoute, verbalisation, vécu, souffrance, parole, lieu d'écoute, etc. Il y a d'ailleurs à cet endroit, une faille, un malentendu entre l'institution du soin et la psychanalyse dont le projet reste de transformer la misère hystérique en malheur banal (Freud, 1895/1975, p. 247).

L'homme hypermoderne voit ses conditions changer à nouveau. Porté par son aspiration à l'autonomie, il se retrouve soumis à une nouvelle forme de contrainte:l'autonomie comme condition. Certes tout lui est permis mais il est redevable de tout: de ses réussites et de ses échecs. A. Ehrenberg (2010) parle de la figure de l'entrepreneur imposée à tous et pas seulement dans le champ du travail: chacun devient l'entrepreneur de sa propre vie (p. 217), chacun engrange à titre individuel réussite mais aussi échec (p. 210). Dans ce contexte, le thérapeute ${ }^{5}$ devient l'autre figure du monde hypermoderne, (p. 218): celui qui dope les capacités individuelles et qui répare les dégâts en particulier au travail (burn-out, harcèlement, chômage, exclusion). C'est ainsi qu'émerge la notion de «souffrance sociale» et en corollaire une clinique psychosociale débouchant sur une psychologisation de la société ou plus précisément, sur un traitement psychologique de la question sociale. M. Otero (2003), sociologue québécois, donne dans ce contexte une fonction de régulation aux interventions psychosociales et psychothérapeutiques. On peut évoquer également les travaux d'auteurs belges: J. De Munck et coll. (2003) et D. Vancken et C. Macquet (2006), ou encore l'intéressante analyse de D. Fassin (2006) en France.

A. Ehrenberg (2004) met de l'avant le second glissement de la psychiatrie qui, après avoir été étendue à la souffrance psychique (cf. supra), se voit 
rebaptisée santé mentale (Ehrenberg, 2004), ouvrant à son extension à l'infini. R. Gori (2006, 2008; Gori et Del Vogo, 2008) dénonce comme nouvelle forme de contrôle social, cette transformation de la psychiatrie qui normalise tous les comportements (et rend pathologique tout écart à la norme ${ }^{6}$ ).

Il ne faudrait pourtant pas voir la postmodernité et l'hypermodernité comme deux temps chronologiques créant dans leur sillage deux problématiques psychopathologiques différenciées. Elles renvoient en fait à deux postures conjointes débouchant sur une injonction paradoxale. La culture impose une nouvelle loi à la fois permissive (tu peux tout, tu as droit à tout) et répressive selon une modalité particulière qui est celle de la responsabilité: de ta santé, de tes échecs, de tes troubles mentaux, etc. C'est bien dans cet agencement entre permissivité et responsabilité que se jouent les nouvelles formes de contraintes sociales. R. Castel (1981) parlait déjà de cette nouvelle ère où nous aurions à nous programmer nous-mêmes.

L'institution du soin devient le lieu des nouvelles formes de régulation et de contrôle social. Il s'agit de limiter les effets cumulatifs tant des excès liés à la revendication au bonheur (qu'il s'agisse d'excès en moins — dépression ou en plus - addiction) que des déstructurations liées à une (dés)organisation sociale anomique. Dans cette nouvelle figure du psy comme contrôleur, régulateur, réparateur, la constellation symbolique du soin bascule vers un soin normatif et efficace.

Mais à l'heure du soin performant, une place pour la psychanalyse est-elle encore possible? Est-elle souhaitable? Ou, dit autrement, et pour reprendre une question de J.B. Pontalis relayée par A. Ehrenberg (2014) : que peut la psychanalyse et quelles en sont les limites?

R. Kaës répond que certes la psychanalyse doit «par la mise en œuvre de nouveaux dispositifs, traiter les souffrances et les pathologies générées » par les changements de la condition anthropologique», que "sa réflexion sur les processus de formation de la subjectivité dans le monde contemporain est indispensable», mais que «les solutions au malêtre relèvent aussi d'autres instances, du projet politique tout d'abord et de son pouvoir sur l'économie et la culture» $(2012$, p. 31$)$.

À l'évidence, l'auteur ne place pas la psychanalyse en position de toute puissance et renvoie clairement au champ du politique ce qui lui revient. Il s'agit, toujours selon R. Kaës, de prendre en considération «la différence et les articulations entre le sujet social et le sujet de l'inconscient» (p. 116) sans que, rajouterions-nous, le sujet de l'inconscient ne soit passé par pertes et profits (Benasayag, 2015), ni que le sujet social en soit réduit à un sujet 
psychologique. Défendre la place de la psychanalyse dans l'institution du soin devient alors un acte de résistance à l'entreprise de transformation du sujet en homo psychologicus comme en avait eu l'intuition J. Lacan dès 1951. Une façon aussi de lutter contre la mise à mal de l'instituant au profit d'un organisationnel toujours plus puissant. Mouvement largement représenté par la figure emblématique de R. Gori.

Mais la psychanalyse ne peut se contenter d'être un porte-drapeau du sujet. Elle ne peut et ne doit pas se faire idéologie. La politique est affaire de citoyens, fussent-ils psychanalystes, mais pas de la psychanalyse qui, comme le dit R. Kaës, ne peut tout résoudre (voir ci-dessus). Ses objectifs sont de «penser d'autres dispositifs et s'interroger tant au niveau épistémologique que méthodologique sur l'extension de ses pratiques et de ses théorisations» (Kaës 2012, p. 116). Ce chantier s'est ouvert dans les années 1940 avec les travaux sur les états-limites et les psychoses. Travaux initiés selon nous par W. R. Bion (contenance) et D. Winnicott (transitionnalité) qui ont abouti à un changement de paradigme au sein de la psychanalyse, la faisant passer d'une théorisation des contenus à une théorisation des contenants de pensées. R. Roussillon (2008, p. 6) parle d'ailleurs de coupure épistémologique au sein de la psychanalyse à partir de l'œuvre de D. Winnicott.

L'enjeu fondamental de cette nouvelle psychanalyse concerne la fonctionnalité du préconscient. Le préconscient est l'instance qui permet la transformation des contenus de pensées, qui donne à la psyché sa capacité associative et interprétative (Kaës, 2012, p. 168). C'est aussi ce qui va donner accès à la réflexivité portée par une triple composante: se sentir, se voir et s'entendre (Roussillon 2008, p. 8). La dernière composante pouvant être reliée à la capacité narrative, support de l'identité narrative (Ricoeur, 1985).

Freud avait bien conceptualisé le travail du préconscient grâce à sa théorisation du travail du rêve, mais il en faisait d'emblée un processus intrapsychique et fonctionnel. Les notions de contenance ou de transitionnalité mettent en évidence que cette fonctionnalité résulte de processus liés à la fois au corps et à l'autre ${ }^{7}$, avec ce que cela implique d'entraves et d'échecs.

Il s'agit alors de réfléchir à des dispositifs thérapeutiques propres à instaurer ces processus, mais également à les restaurer quand ils ont été mis à mal par des situations ou des événements désubjectivants. Le but est de rendre au préconscient sa valence fonctionnelle permettant d'ouvrir progressivement au champ de la parole, de (r)établir une capacité narrative et la temporalité qui lui est liée (passé et avenir). Dit autrement, il s'agit d'instaurer/restaurer 
une aire d'illusion primaire qui autorise la reprise du travail de désillusionnement sans effondrement ni forçage (Roussillon 2008, p. 17).

Cette question aurait pu être traitée à partir des écrits d'A. Green sur les processus tertiaires $(1995,2002)$. Tout en reconnaissant l'apport fondamental de ce concept, nous avons opté pour les travaux sur l'analyse transitionnelle qui offrent une approche plus fonctionnelle de ces processus. Nous considérons d'ailleurs l'analyse transitionnelle comme une explicitation des fondements intersubjectifs de ce que A. Green a voulu souligner avec ce concept. Cette notion est due initialement à R. Kaës $(1976,1979)$ qui l'élabore dans le cadre du groupe. D. Anzieu (1979) la reprendra pour évoquer une thérapie individuelle avant d'en poursuivre la conceptualisation du côté du moi-peau avec la notion d'enveloppes psychiques (Anzieu 1994; Anzieu et Haag, 1993). R. Roussillon (2008) va reprendre la notion d'analyse transitionnelle et la conceptualiser à partir des trois fonctions du cadre définies par P. Delion (2000, 2001a, 2001b) dans le champ de la psychothérapie institutionnelle: fonctions phorique, sémaphorique et métaphorique.

\section{Fonction phorique}

P. Delion considère la fonction phorique comme une traduction de la notion de holding de Winnicott et la renvoie à la fonction d'accueil. Elle lui a été inspirée par les romans de Michel Tournier, en particulier, Le roi des aulnes (Delion, 2012). Il écrit que la fonction phorique revient à «proposer un espace physique mais surtout psychique dans lequel ce qui est non-lieu va pouvoir devenir événement. Là seulement peut s'opérer le début d'une temporalisation transférentielle» (2005, p. 111).

Cette idée est reprise par R. Roussillon pour qui «la fonction phorique du dispositif "sémaphorise" potentiellement l'histoire de la rencontre avec les objets porteurs d'une fonction symbolique pour l'analysant, l'histoire de la manière dont ils ont, ou n'ont pas, occupé cette fonction » (2008, p. 29).

Conception qu'on retrouve aussi chez R. Kaës avec les fonctions de contenance et de conteneur: "J'appelle contenance la capacité d'héberger en soi (soi pouvant être un sujet ou un groupe, une famille ou une institution) des formations psychiques appartenant à un autre sujet ou à plusieurs autres sujets. [...] La contenance est l'acceptation non toxique et non fusionnelle d'un lien "entre ce qui est déposé, celui qui dépose et le dépositaire", selon le schéma de E. Pichon-Rivière réélaboré par J. Bleger» (2012, p. 164).

Nous illustrerons cette première fonction par un petit exemple d'ordre institutionnel qui nous a semblé intéressant car il montre que la fonction 
phorique n'est pas que l'affaire du clinicien mais de l'institution dans son ensemble; si la première enveloppe est trouée, rien d'autre du processus ne pourra advenir.

L'une de mes patientes demande un rendez-vous de première consultation dans un $\mathrm{CMPP}^{8}$ pour son fils âgé de trois ans. Ma patiente indique, quand on lui demande ce qui motive la consultation, que celle-ci fait suite à des problèmes de violence conjugale. Il lui est alors demandé s'il y a eu plainte. Interloquée Mélanie ${ }^{9}$ répond qu'elle a été classée sans suite.

On lui rétorque alors: «Ben dans ce cas, pas la peine de le noter sur le motif de consultation.»

Certes, il y a lieu de s'interroger sur ce qui a pu motiver Mélanie à se présenter sous ce libellé alors que les violences conjugales remontent à plusieurs années (le couple s'est séparé peu après la naissance de l'enfant suite à ces épisodes de violence).

Travail qu'on ne manquera pas de faire toutes les deux en séance. N'empêche, la question demeure entière de l'accueil qui a pu lui être fait. Il se rejoue en effet dans ce moment toute la problématique de Mélanie la conduisant répétitivement à se voir disqualifiée dans ses ressentis : tout d'abord par sa famille, mais aussi par le psychiatre qu'elle a rencontré suite à une tentative de suicide, ou encore par les policiers auprès de qui elle viendra déposer plainte après les violences subies de la part de son conjoint.

Instantané qui illustre à merveille la fonction oméga [en opposition à la fonction alpha] «reproduisant pour le sujet, le type de relation qu'il a instauré précédemment dans sa famille» (Willians, 1997, cité par Delion, 2005, p. 111).

\section{Fonction sémaphorisante}

Tous les auteurs s'accordent sur le fait que la fonction phorique est un processus sémaphorisant même si leurs conceptualisations peuvent varier. Pour P. Delion, «les traces ne peuvent se faire signe et c'est à celui qui les reçoit de les transformer en signes propres à entrer dans le processus de symbolisation» (2005, p. 112). Propos dans lesquels on retrouve la notion de transformation liée chez R. Kaës à la fonction de conteneur qu'il commence à élaborer dès 1976: «Toutefois la contenance passive ne suffit pas. Le dépositaire (et ici encore la psyché maternelle en est le prototype) a pour objectif de 
créer un contenant et un cadre capables d'accueillir avec souplesse et bienveillance ces objets incontrôlables et détériorés. C'est ici que la contenance se convertit en fonction conteneur: j'ai attribué à cette fonction le processus de transformation des contenus par la capacité de contenance» (Kaës, 2012, p. 164). Cette notion de conteneur sera reprise par D. Anzieu (1986, 1990) qui, comme l'indique A. Ciccone (2001), en inversera les termes, désignant par contenant ce qui revient au conteneur et vice versa (Anzieu, 1994; Anzieu et Haag, 1993). R. Roussillon (2008) opte pour la terminologie de P. Delion mais en marque la dimension processuelle en parlant de fonction sémaphorisante (et métaphorisante).

Benoît est venu me voir car il sent une grande violence monter en lui et en a peur: «Le sport ça suffit plus, je sens que je vais disjoncter.» À le voir, le risque est bien réel, on le sent prêt à exploser (il a failli agresser un conducteur lors d'une altercation en voiture). Il a choisi un métier d'action qui lui a permis de canaliser cette violence mais il sent que cela ne tient plus. Il ne sait pas comment commencer les séances, il ne «sait pas quoi dire»; pourtant sa vie comme celle des générations qui le précèdent est émaillée de nombreux secrets et traumatismes.

Il me faudra alors, en début de séance, proposer les premiers mots, faire le lien avec les séances précédentes, le relancer sur des narrations laissées en suspens. Lui renvoyer régulièrement toute la portée traumatique de ce qu'il me et se révèle par lambeaux au cours des séances.

Il dira que les avant-séances le mettent en tension, qu'il les appréhende mais que cela lui fait du bien de parler. Il n'a d'ailleurs plus d'eczéma (j’apprendrai alors qu'il a été un grand eczémateux enfant). Ce qui laisse penser que les premières enveloppes narratives ont été défaillantes, qu'il reste avec une peau psychique à vif. On peut penser d'ailleurs que s'il appréhende les séances c'est qu'il en ressent la dimension potentiellement excitante, irritante (eczéma), mais qu'il expérimente en cours de séance que l'enveloppe narrative peut aussi s'avérer apaisante. Benoît découvre progressivement que les mots peuvent faire signe... qu'ils peuvent être accueillis et contenus par l'autre. Il découvre parallèlement qu'il peut parler, penser, mettre en image ses sensations, en récit son histoire. Il va pouvoir se dégager d'une mère excitante imposant un corps à corps dont il ne peut se dégager que par la motricité et expérimenter la rencontre entre deux subjectivités, deux subjectivités en lien mais différenciées. 
R. Roussillon conceptualise la fonction sémaphorisante à partir de la double transitionnalité de $\mathrm{D}$. Winnicott: celle de l'objet transitionnel et celle de la transitionnalité intersubjective qui se constitue dans le lien à la mère. Il souligne qu'il s'agit d'un processus transitif où l'objet ouvre sur le jeu qui ouvre sur l'autre-sujet. Transitivité dont il rend compte grâce à un néologisme: l'objeu. La psyché aurait besoin d'un détour par l'objet pour accéder à la fonction de représentation, mais le processus ne pourrait advenir que si l'objet est support de jeu. R. Roussillon se réfere au concept de médiummalléable de M. Milner (1979) pour qui jouer revient à une déformation/ transformation de la matière jusqu'à la représentation. Mais l'objet ne peut se faire transitionnel, devenir objeu que s'il est accompagné par l'autre-sujet (maternel, thérapeutique). Celui-ci doit porter la valeur signifiante du comportement du sujet, lui donner un sens potentiel ce qui implique, toujours selon R. Roussillon et en référence à D. Winnicott (1971), que l'Autre Sujet doit accepter d'être attaqué par la pulsionnalité de l'enfant et montrer qu'il y survit.

Coralie, pourtant intelligente et cultivée, ne sait pas jouer avec les mots, ne sait pas les triturer, les déformer, ni seule, ni avec l'autre. Pas de squiggle narratif possible (Proia-Lelouey 2013), les propos de l'autre ne sont pas perçus sur une modalité sémaphorisante mais sur un mode performatif et injonctif conduisant à des vécus paranoïdes intenses. Qu'est-ce qui de la fonction maternelle a pu être défaillant? Les vécus transféro-contretransférentiels de dépression intense laissent envisager une dépression maternelle (Green, 1983) ne laissant aucun espace à l'objeu. Coralie ne peut jouer avec sa mère indisponible ni se jouer de sa mère, sous peine d'effondrement mutuel. Elle reste, dans un temps suspendu, accrochée au regard, aux mots de l'autre comme le noyé à son sauveteur. Impossible de lâcher prise ni d'expérimenter la mise en langage, en récit, seule en présence de l'analyste.

\section{Fonction métaphorisante}

L'accès à la fonction métaphorisante rend compte d'une psyché restaurée, d'un préconscient fonctionnel autorisant la capacité à jouer avec le langage, à penser seul en présence de l'autre c'est-à-dire ouvrant à l'intrasubjectif et à la réflexivité. $\mathrm{R}$. Roussillon y met deux conditions: que l'autre devienne "un autre-sujet, un sujet semblable, double de soi», mais aussi que «le sujet reconnaisse et s'approprie, dans ce qui lui est réfléchi, ce qui 
le concerne spécifiquement, le différencie de l'autre-sujet» (2008, p. 34-35). C'est ce double mouvement, qui, précise encore l'auteur, permet de rencontrer, dans la relation intersubjective, la différence, ouvrant à l'auto-subjectif, à l'intra-subjectif.

Mélanie s'émerveille en séance de pouvoir jouer avec sa pensée: «ça marche de mieux en mieux» dit-elle. Elle peut ainsi progressivement, durant les séances, se vivre autrement qu'en double collé de l'autre-maternel et s'ouvrir à une dynamique odipienne conflictuelle mais non destructrice. Cependant, le «lien impossible» maintenu par nécessité avec le père de son enfant relance, à chaque conflit, la destructivité. Aussi, ce lien nécessite que patiemment nous parcourions à nouveau (mais de plus en plus aisément) l'ensemble du processus pour remettre en signe et en pensée ce que les empiètements ont pu détruire de cet espace intra-subjectif.

Coralie en revanche, n'est toujours pas arrivée à construire une aire transitionnelle, de transformation et continue à recourir à la solution masochiquenarcissique (Green 1993). Noyau de culpabilité primaire, noyau de malêtre où l'enfant se sent à l'origine de tout ce qui dysfonctionne (Roussillon, 2008, p. 19) et le laisse dans une totale inconsolabilité. C'est toute la capacité de survivance de l'autre-sujet thérapeute qui est alors en jeu...

Nous sommes directement passée de l'analyse de l'institution du soin à une réflexion sur les dispositifs individuels. Nous n'en oublions pas pour autant qu'initialement, R. Kaës a inscrit l'analyse transitionnelle dans le champ des dispositifs de groupe et implicitement, dans celui des dispositifs institutionnels ${ }^{10}$. Nous souscrivons pleinement, comme notre première illustration clinique l'a montré, à l'idée que «chaque espace est soutenu et travaillé par celui ou ceux qui l'encadrent, le contiennent et l'étayent à un niveau logique supérieur» (Kaës, 2012, p. 110). Mais l'auteur va plus loin et souligne que le processus ne s'arrête pas là. Il faut également penser un niveau méta qui englobe et traverse ces cadres, lui-même porté par deux garants: métapsychique et métasocial. Nous pouvons ainsi conclure, pour répondre à Ehrenberg (2014), que si la psychanalyse ne peut intervenir directement sur les cadres métasociaux et les transformations ${ }^{11}$ qu'ils subissent, elle peut, en revanche, théoriser la façon dont ces transformations affectent les garants métapsychiques (Kaës 2012, p. 113).

Nous pouvons, de plus, affirmer que la psychanalyse peut et doit rester au cour de l'institution du soin car elle reste un instrument inégalable de 
désaliénation. Elle permet au sujet de déterminer ce qui, de sa souffrance, lui est imposé par sa configuration psychique personnelle, par les (nouvelles) conditions de la civilisation et par la condition même de sujet civilisé. La psychanalyse lui permettant aussi, une fois les enveloppes psychiques instaurées/restaurées, de disposer d'un espace d'illusion, d'un espace de culture et de création.

\author{
Nadine Proia-Lelouey \\ nadine.proia-lelouey@unicaen.fr
}

\title{
Notes
}

1. Nous renvoyons le lecteur au chapitre 6 de l'ouvrage d'A. Ehrenberg et à sa note 17 où il fait une petite revue des publications sur ce thème initiée par J.-P. Lebrun: Un monde sans limite: essai pour une clinique psychanalytique du social, Érès 1997. Nous pouvons citer en plus F. Richard: L'actuel malaise dans la culture, Éditions de l'Olivier, 2011 et R. Kaës: Le malêtre, Dunod, 2012.

2. La majuscule à Institutions est une manière de marquer la différence entre les Institutions relevant du niveau sociologique (manières collectives de penser et d'agir au niveau de la société en général; en d'autres termes, les grandes institutions essentiellement sous le sceau de l'État) des institutions relevant du niveau psychosociologique (manière collective de penser et d'agir au niveau de certaines pratiques sociales). Si on reprend l'exemple du soin, nous constatons qu'il existe dans les deux registres: comment l'État pense et organise le soin, comment les acteurs sociaux pensent et agissent le soin.

3. Voir aussi M. Otero (2003, 5-42) pour une présentation de ces auteurs.

4. Voir aussi le Moi et le Ça (1923/1991, p. 229).

5. Ou le coach, voir R. Gori et P. Le Coz: L'mpire des coachs. Une nouvelle forme de contrôle social, Albin Michel, 2006.

6. Voir le DSM-V, 2013.

7. Si R. Kaës insiste sur la dimension intersubjective, il précise aussi qu’il importe de réactualiser une autre dimension du préconscient qui est sa dimension cénesthésique, associée au pôle moteur de l'appareil psychique (2012, p. 168) ce qui introduit la question du corps et de l'acte.

8. Centre médico-psycho-pédagogique.

9. Prénom fictif.

10. Concernant ces niveaux, nous renvoyons le lecteur aux contributions des collègues dans ce numéro.

11. Suivant cet auteur nous préférons parler de transformation plutôt que de défaillance.

\section{Références}

Anzieu, D., Haag et G. (1993). Les contenants de pensée. Paris: Dunod.

Anzieu, D. (2004). La démarche de l'analyse transitionnelle en psychanalyse individuelle. Dans R. Kaës (dir.), Crise, rupture et dépassement (3 éd. p. 186-221). Paris: Dunod.

Anzieu, D. (1986). Cadre psychanalytique et enveloppes psychiques. Journal de la psychanalyse de l'enfant, 2, 12-24.

Anzieu, D. (1994). Le penser. Du moi-peau au moi-pensant. Paris: Dunod. 
Anzieu, D. (1990). L’Épiderme nomade et la Peau psychique. Paris: Apsygée.

Benasayag, M. (2015). Clinique du mal-être, la "psy» face aux nouvelles souffrances psychiques. Paris: La Découverte.

Castel, R. (1973). Le psychanalysme. Paris: librairie François Maspéro.

Castel, R. (1981). La gestion des risques. Paris: Minuit.

Castel, R. et Le Cerf J-F. (1980a). Le phome psy et la socifranise. Vers une nouvelle culture psychologique. Le Dat, $\mathrm{n}^{\circ} 1,32-45$.

Castel, R. et Le Cerf J-F. (1980b). Le phome psy et la socifranise. Le Dat, n 2, 39-47.

Castel, R. et Le Cerf J-F. (1980c). Le phome psy et la socifranise. L'après psychanalyse (fin). Le Dat, $\mathrm{n}^{\circ} 3,22-30$.

Ciccone, A. (2001). Enveloppe psychique et fonction contenante: modèles et pratiques. Cahiers de psychologie clinique, 17 (2), 81-102.

De Munck, J. et coll. (2003). Santé mentale et citoyenneté. Gent, Belgique: Academia Press.

Delion, P. (2000). L'enfant autiste, le bébé et la sémiotique. Paris: Presses universitaires de France.

Delion, P. (2001a). Nathanaël, sa psychose et ses institutions. Revue de psychothérapie psychanalytique de groupe, 36 (1), 7-17.

Delion, P. (2001b). Thérapeutiques institutionnelles. Encyclopédie Médico-chirurgicale Psychiatrie. Repéré à www.revue-institutions.com/articles/therapeutiquesinstitution. pdf

Delion, P. (2005). Soigner la Personne psychotique: concepts, pratiques et perspectives de la psychothérapie institutionnelle. Paris: Dunod.

Delion, P. (2012). Donald Winnicott, Michel Tournier et la fonction phorique. Dans A. Braconnier et B. Golse (dir.), Winnicott et la création humaine (p. 17-35). Toulouse: Érès.

Ehrenberg, A. (1995). L'individu incertain. Paris: Calman Lévy.

Ehrenberg, A. (1998). La fatigue d'être soi. Paris: Odile Jacob.

Ehrenberg, A. (2004). Remarques pour éclaircir le concept de santé mentale. Point de vue. Revue française des affaires sociales, $\mathrm{n}^{\circ} 1,77-88$.

Ehrenberg, A. (2010). La société du malaise. Paris: Odile Jacob.

Ehrenberg, A. (2011). La société du malaise: Une présentation pour un dialogue entre clinique et sociologie. Adolescence, 77 (3), 553-570.

Ehrenberg, A. (2014). Narcissisme, individualisme, autonomie: malaise dans la société? Revue française de psychanalyse, 78 (1), 98-109.

Fassin, D. (2006). Souffrir par le social, gouverner par l'écoute. Une configuration sémantique de l'action publique. Politix, 73 (1), 137-157.

Freud, S. (1916). Passagèreté. CEuvres complètes, XIII. Paris: Presses universitaires de France, 1988.

Freud, S. (1923). Le Moi et le Ça. Euvres complètes, XVI. Paris: Presses universitaires de France, 1991.

Freud, S. (1930). Le malaise dans la culture. Euvres complètes, XVIII. Paris: Presses universitaiers de France, 1994.

Freud, S. (1940). L'Abrégé de psychanalyse. Euvres complètes, XX. Paris: Presses universitaires de France, 2010.

Freud, S. et Breuer, J. (1895). Études sur l'hystérie. Paris: Presses universitaires de France, 1975.

Gauchet, M. (2002). La démocratie contre elle-même. Paris: Gallimard.

Gauchet, M. (2002). La religion dans la démocratie: parcours de la laïcité. Paris: Gallimard.

Gori R. (2006). La construction du trouble comme entreprise de normalisation. La lettre de l'enfance et de l'adolescence, 66 (4), 31-41. 
Gori R. (2008). Malaise dans la psychiatrie? Cliniques méditerranéennes, 78 (2), 49-64.

Gori R. et Del Volgo M.J. (2008). Exilés de l'intime. La médecine et la psychiatrie au service du nouvel ordre économique. Paris: Denoël.

Gori R. et Le Coz P. (2006). L'empire des coachs. Une nouvelle forme de contrôle social. Paris: Albin Michel.

Green, A. (1983). La mère morte. Dans Narcissisme de vie, narcissisme de mort (p. 222-253). Paris: Minuit.

Green, A. (1993). Le travail du négatif. Paris: Minuit.

Green, A. (1995). Annexe D, notes sur les processus tertiaires. Dans Propédeutique (p. 151155). Seyssel, France: Champs Vallon.

Green, A. (2002). De la tiercéité. Dans La pensée clinique (p. 259-308). Paris: Odile Jacob.

Juan, S. (2006). Le combat de l'Organisation et de l'Institution, SociologieS [En ligne], Théories et recherches, repéré à http://sociologies.revues.org/index582.html

Kaës, R. (1979). Crise, rupture et dépassement. Paris: Dunod, 2004.

Kaës, R. (2012). Le malêtre. Paris: Dunod.

Kaës, R. (1976). Analyse intertransférentielle, fonction alpha et groupe conteneur. L'Évolution psychiatrique, 41 (2), 1976, 239-247.

Lacan, J. (1952). Intervention sur le transfert. Revue française de psychanalyse, 16 (1-2), 154-163.

Lasch, C. (1981). Le complexe de narcissisme, la nouvelle sensibilité américaine. Paris: Lafond. Lipovetsky, G. (1983). L'ère du vide, essai sur l'individualisme contemporain. Paris: Gallimard.

Milner, M. (1979). Le rôle de l'illusion dans la formation du symbole, Revue de psychanalyse, $\mathrm{n}^{\text {os }} 5-6,844-874$.

Otero, M. (2003). Les règles de l'individualité contemporaine, santé mentale et société. Québec: Presses de l'Université de Laval.

Proia-Lelouey, N. (2012). L'entretien en psychologie. Paris: In Press.

Proia-Lelouey, N. (2013). Les premiers entretiens: une aire intermédiaire. Santé mentale, $\mathrm{n}^{\circ} 176,28-32$.

Rabant, C. (2010). Embobiner la pulsion de mort. Che vuoi? 33 (1), 63-74.

Richard, F. (2011). L'actuel malaise dans la culture. Paris: Éditions de l'Olivier.

Ricoeur, P. (1985). Temps et récits III, Le temps raconté. Paris: Seuil.

Roudinesco, E. (2005). Mais pourquoi tant de haine? Paris: Navarin.

Roussillon, R. (2008). Le transitionnel, le sexuel et la réflexivité. Paris: Dunod.

Turmel, A. (1997). Le retour du concept d'institution. Dans A. Turmel (dir.), Culture, institution et savoir. Culture française d'Amérique (p. 1-24). Québec; Les Presses de l'Université Laval. URL: http://www.uqac.uquebec.ca/zone30/Classiques_des_sciences_sociales/ index. html

Vancken, D. et Macquet, C. (2006), Vers une psychologisation de la société. Paris: Belin.

Winnicott, D.W. (1971). L'utilisation de l'objet et le mode de relation à l'objet au travers des identifications. Dans Jeu et réalité (p. 91-119). Paris: Gallimard, 1975. 